\title{
Feedback Stabilization of Uncertain Systems Using a Stochastic Digital Link
}

\author{
Nuno C. Martins, Munther A. Dahleh and Nicola Elia
}

\begin{abstract}
We study the stabilizability of uncertain systems in the presence of finite capacity feedback. Motivated by the structure of communication networks, we consider a stochastic digital link that sends words whose size is governed by a random process. Such link is used to transmit state measurements between the plant and the controller. We derive necessary and sufficient conditions for the internal and the external stabilizability of the feedback loop. In accordance with previous publications, stabilizability of unstable plants is possible if and only if the link's average transmission rate is above a positive critical value. In our formulation the plant and the link can be stochastic. In addition, stability in the presence of uncertainty in the plant is analyzed using a small-gain argument. We show that the critical average transmission rate, for stabilizability, depends on the description of uncertainty and the statistical properties of the plant as well as the link.
\end{abstract}

\section{INTRODUCTION}

With a wide range of formulations, control in the presence of communication constraints has been the focus of intense research. The need to remotely control one or more systems from a central location, has stimulated the study of stabilizability of unstable plants when the information flow in the feedback loop is finite. Such limitation results from the use of an analog communication channel or a digital link as a way to transmit information about the state of the plant. It can also be the abstraction of computational constraints created by several systems sharing a common decision center.

Various publications in this field have introduced necessary and sufficient conditions for the stabilizability of unstable plants in the presence of data-rate constraints. The construction of a stabilizing controller requires that the data-rate of the feedback loop is above a non-zero critical value [17], [18], [14], [15], [8]. Different notions of stability have been investigated, such as containability [21], [22], moment stability [15] and stability in the almost sure sense [17]. The last two are different when the state is a random variable. That happens when disturbances are random or if the communication link is stochastic. In [17] it is shown that the necessary and sufficient condition for almost sure stabilizability of finite dimensional linear and time-invariant systems is given by an inequality of the type $\mathcal{C}>\mathcal{R}$. The parameter $\mathcal{C}$ represents the average data-rate of the feedback loop and $\mathcal{R}$ is a quantity that depends on the eigenvalues of $A$, the dynamic matrix of the system.

Nuno C. Martins and Munther A. Dahleh are at LIDS, Dept. of EECS, Massachusetts Institute of Technology nmartins @mit.edu, dahleh@mit.edu

Nicola Elia is with the Dept. of Electrical Engineering a the Iowa State University nelia@iowastate.edu
If a well defined channel is present in the feedback loop then $\mathcal{C}$ may be taken as the Shannon Capacity. If it is a digital link then $\mathcal{C}$ is the average transmission rate. Different notions of stability may lead to distinct requirements for stabilization. For tighter notions of stability, such as in the m-th moment sense, the knowledge of $\mathcal{C}$ may not suffice. More informative notions, such as higher moments or anytime capacity [15], are necessary. In general, $\mathcal{C}>\mathcal{R}$ is not sufficient for stabilization in the $\mathrm{m}$-th moment sense. This conclusion was derived in [16], where sufficiency was stated in terms of Anytime Capacity. Our work is largely motivated by [16].

In this paper, we study the moment stabilizability of uncertain systems in the presence of a stochastic digital link. In contrast with [13], we consider systems whose timevariation is governed by an identically and independently distributed (i.i.d.) process which may be defined over a continuous and unbounded alphabet. We also provide complementary results to [13], [4], [5], [7] because we use a different problem formulation where we consider external disturbances and uncertainty on the plant and a stochastic digital link. Our work provides a unified framework for robust stabilizability by determining the average transmission rate $\mathcal{C}$ which is necessary to compensate for randomness both in the plant and the digital link. It also shows that larger uncertainty in the plant can be tolerated at the expense of higher $\mathcal{C}$. All of our conditions for stability are expressed as simple inequalities where the terms depend on the description of uncertainty in the plant as well as the statistics of the system and the digital link. A different approach to deal with robustness, with respect to transmission rates, can be found in [9].

In order to focus on the fundamental issues and keep clarity, we derive sufficient conditions only for first order linear systems. According to [11], the extension to a class of finite-dimensional linear systems can be achieved by means of real Jordan forms. The first use of real Jordan forms in this framework was suggested by [18]. It is important to emphasize that our results cannot be extended to arbitrary multi-state linear stochastic systems. As pointed out in [13], non-commutativity creates difficulties in the study of arbitrary time-varying stochastic systems. Results for the fully-observed Markovian case over finite alphabets, in the presence of a deterministic link, can be found in [13].

Besides the introduction, the paper has 3 sections: section II comprises the problem formulation and preliminary definitions; in section III we prove sufficiency conditions by constructing a stabilizing feedback scheme and section 
IV contains the proof of the necessary condition.

The following notation is adopted:

Whenever that is clear from the context we refer to a sequence of real numbers $x(k)$ simply as $x$. In such cases, we may add that $x \in \mathbb{R}^{\infty}$. Random variables are represented using boldface letters, such as $\mathbf{w}$. If $\mathbf{w}(k)$ is a stochastic process, then we use $w(k)$ to indicate a specific realization. According to the convention used for sequences, we may denote $\mathbf{w}(k)$ just as $\mathbf{w}$ and $w(k)$ as $w$. The expectation operator over $\mathbf{w}$ is written as $\mathcal{E}[\mathbf{w}]$. If $E$ is a probabilistic event, then its probability is indicated as $\mathcal{P}(E)$. We write $\log _{2}($.$) simply as \log ($.$) . If x \in \mathbb{R}^{\infty}$, then

$$
\begin{aligned}
\|x\|_{1} & =\sum_{i=0}^{\infty}|x(i)| \\
\|x\|_{\infty} & =\sup _{i \in \mathbb{N}}|x(i)|
\end{aligned}
$$

Definition 1.1: Let $\varrho \in \mathbb{N}_{+} \bigcup\{\infty\}$ be an upper-bound for the memory horizon of an operator. If $G_{f}: \mathbb{R}^{\infty} \rightarrow \mathbb{R}^{\infty}$ is a causal operator then we define $\left\|G_{f}\right\|_{\infty(\varrho)}$ as:

$$
\left\|G_{f}\right\|_{\infty(\varrho)}=\sup _{k \geq 0, x \neq 0} \frac{\left|G_{f}(x)(k)\right|}{\max _{j \leq k}\{|x(j-\varrho+1)|, \ldots,|x(j)|\}}
$$

Note that $\left\|G_{f}\right\|_{\infty(\infty)}$ is just the infinity induced norm of $G_{f}$ :

$$
\left\|G_{f}\right\|_{\infty(\infty)}=\left\|G_{f}\right\|_{\infty}=\sup _{x \neq 0} \frac{\left\|G_{f}(x)\right\|_{\infty}}{\|x\|_{\infty}}
$$

\section{PROBLEM FORMULATION}

We study the stabilizability of uncertain stochastic systems under communication constraints. We consider the following class of stochastic links:

Definition 2.1: (Stochastic Link) Consider a link that, at every instant $k$, transmits $\mathbf{r}(k)$ bits. We define it to be a stochastic link, provided that $\mathbf{r}(k) \in\left\{r_{\min }, \ldots, \bar{r}\right\}$ is a random process satisfying:

$$
\mathbf{r}(k)=\mathcal{C}-\mathbf{r}^{\delta}(k)
$$

where $\mathbf{r}^{\delta}(k)$ is an i.i.d. zero-mean process. More specifically, the link is a stochastic truncation operator $\mathcal{F}_{k}^{l}$ : $\{0,1\}^{\bar{r}} \rightarrow \bigcup_{i=0}^{\bar{r}}\{0,1\}^{i}$ defined as:

$$
\mathcal{F}_{k}^{l}\left(b_{1}, \ldots, b_{\bar{r}}\right)=\left(b_{1}, \ldots, b_{\mathbf{r}(k)}\right)
$$

where $b_{i} \in\{0,1\}$.

Our definition of stochastic link is a generalization of the classical erasure channel. It allows for the study of the more realistic case where communication is performed at a nominal rate and is affected by an uncertain fluctuation. In [11], this definition is motivated as the model of the utilization of a wireless link between two nodes, which may correspond to the plant and a central station.

Given $x(0) \in\left[-\frac{1}{2}, \frac{1}{2}\right]$ and $\bar{d} \geq 0$, we consider nominal systems of the form:

$$
\mathbf{x}(k+1)=\mathbf{a}(k) \mathbf{x}(k)+\mathbf{u}(k)+\mathbf{d}(k)
$$

with $|\mathbf{d}(k)| \leq \bar{d}$ and $\mathbf{x}(i)=0$ for $i<0$.

\section{A. Description of Uncertainty in the Plant}

Let $\varrho \in \mathbb{N}_{+} \bigcup\{\infty\}, \bar{z}_{f} \in[0,1)$ and $\bar{z}_{a} \in[0,1)$ be given constants, along with the stochastic process $\mathbf{z}_{a}$ and the operator $G_{f}: \mathbb{R}^{\infty} \rightarrow \mathbb{R}^{\infty}$ satisfying:

$$
\begin{gathered}
\left|\mathbf{z}_{a}(k)\right| \leq \bar{z}_{a} \\
G_{f} \text { causal and }\left\|G_{f}\right\|_{\infty(\varrho)} \leq \bar{z}_{f}
\end{gathered}
$$

Given $x(0) \in\left[-\frac{1}{2}, \frac{1}{2}\right]$ and $\bar{d} \geq 0$, we study the existence of stabilizing feedback schemes for the following perturbed plant:

$\mathbf{x}(k+1)=\mathbf{a}(k)\left(1+\mathbf{z}_{a}(k)\right) \mathbf{x}(k)+\mathbf{u}(k)+\mathbf{z}_{f}(k)+\mathbf{d}(k)$

where the perturbation processes $\mathbf{z}_{a}$ and $\mathbf{z}_{f}=G_{f}(\mathbf{x})$ satisfy (5)-(6). Notice that $\mathbf{z}_{a}(k)$ may represent uncertainty in the knowledge of $\mathbf{a}(k)$, while $\mathbf{z}_{f}(k)$ is the output of the feedback uncertainty block $G_{f}$. We chose this structure because it allows the representation of a wide class of model uncertainty. It is also allows the construction of a suitable stabilizing scheme.

Example 2.1: If $\mathbf{z}_{f}(k)=\mu_{0} \mathbf{x}(k)+\ldots+\mu_{n-1} \mathbf{x}(k-n+1)$ then $\left\|G_{f}\right\|_{\infty(\varrho)}=\sum\left|\mu_{i}\right|$ for $\varrho \geq n$.

In general, the operator $G_{f}$ may be nonlinear and timevarying.

\section{B. Statistical Description of $\mathbf{a}(k)$}

The process $\mathbf{a}(k)$ is i.i.d. and independent of $\mathbf{r}(k)$ and $x(0)$, meaning that it carries no information about the link nor the initial state. In addition, it satisfies:

$$
\log (|\mathbf{a}(k)|)=\mathcal{R}+\mathbf{l}^{\delta}(k)
$$

where $\mathbf{l}^{\delta}(k)$ is a zero mean and i.i.d. sequence. Since $\mathbf{a}(k)$ is ergodic, we also assume that $\mathcal{P}(a(k)=0)=0$, otherwise the system is trivially stable. Such assumption is also realistic if we assume that (7) comes from the discretization of a continuous-time system.

\section{Functional Structure of the Feedback Interconnection}

In the subsequent text we describe the feedback loop structure, which might also be designated as information pattern [20]. Besides the plant, there are two blocks denoted as encoder and controller which are stochastic operators. At any given time $k$, we assume that both the encoder and the controller have access to $a(0), \ldots, a(k)$ and $r(k-1)$ as well as the constants $\varrho, \bar{z}_{f}, \bar{z}_{a}$ and $\bar{d}$. The encoder and the controller are described as:

- The encoder is a function $\mathcal{F}_{k}^{e}: \mathbb{R}^{k+1} \rightarrow\{0,1\}^{\bar{r}}$ that has the following dependence on observations:

$$
\mathcal{F}_{k}^{e}(x(0), \ldots, \mathbf{x}(k))=\left(\mathbf{b}_{1}, \ldots, \mathbf{b}_{\bar{r}}\right)
$$

- The control action results from a map, not necessarily memoryless, $\mathcal{F}_{k}^{c}: \bigcup_{i=0}^{\bar{r}}\{0,1\}^{i} \rightarrow \mathbb{R}$ exhibiting the following functional dependence:

$$
\mathbf{u}(k)=\mathcal{F}_{k}^{c}(\overrightarrow{\mathbf{b}}(k))
$$


where $\vec{b}(k)$ are the bits successfully transmitted though the link, i.e.:

$$
\overrightarrow{\mathbf{b}}(k)=\mathcal{F}_{k}^{l}\left(\mathbf{b}_{1}, \ldots, \mathbf{b}_{\bar{r}}\right)=\left(\mathbf{b}_{1}, \ldots, \mathbf{b}_{\mathbf{r}(k)}\right)
$$

As such, $\mathbf{u}(k)$ can be equivalently expressed as

$$
\mathbf{u}(k)=\left(\mathcal{F}_{k}^{c} \circ \mathcal{F}_{k}^{l} \circ \mathcal{F}_{k}^{e}\right)(x(0), \ldots, \mathbf{x}(k))
$$

Definition 2.2: ( Feedback Scheme) We define a feedback scheme as the collection of a controller $\mathcal{F}_{k}^{c}$ and an encoder $\mathcal{F}_{k}^{e}$.

\section{Further remarks on our assumptions}

We would like to emphasize that our sufficiency conditions hold under the assumptions of section II-C. In real applications, the knowledge of $r(k-1)$ by the decoder and the encoder requires a synchronization scheme [11], which relies on the use of an acknowledge signal as suggested by [18], [16]. Another aspect is the fact that erasure occurs on the least important bits, i.e., it performs a truncation. This assumption makes sense in the wireless communication between two nodes [11], where packets are sent sequentially. The wireless communication works asynchronously with the plant and the controller, so that the number of packets successfully sent is a random variable which depends on collisions and fading. Erasure of packets in random positions is characteristic of large networks where the packets travel through different routers. The necessary condition is general and does not depend on the knowledge of $r(k-1)$ as well as the position of erasure.

\section{E. Problem Statement and M-th Moment Stability}

Definition 2.3: (Worst Case Envelope) Let $\mathbf{x}(k)$ be the solution to (7) under a given feedback scheme. Given any realization of the random variables $\mathbf{r}(k), \mathbf{a}(k), \mathbf{z}_{f}(k), \mathbf{z}_{a}(k)$ and $\mathbf{d}(k)$, the worst case envelope $\overline{\mathbf{x}}(k)$ is the random variable whose realization is defined by:

$$
\bar{x}(k)=\sup _{x(0) \in\left[-\frac{1}{2}, \frac{1}{2}\right]}|x(k)|
$$

Consequently, $\overline{\mathbf{x}}(k)$ is the smallest envelope that contains every trajectory generated by an initial condition in the interval $x(0) \in\left[-\frac{1}{2}, \frac{1}{2}\right]$. We adopted the interval $\left[-\frac{1}{2}, \frac{1}{2}\right]$ to make the paper more readable. All results are valid if it is replaced by any other symmetric bounded interval.

Our problem consists in determining necessary and sufficient conditions that guarantee the existence of a stabilizing feedback scheme. The results must be derived for the following notion of stability.

Definition 2.4: (m-th Moment Robust Stability) Let $m>0, \varrho \in \mathbb{N}_{+} \bigcup\{\infty\}, \bar{z}_{f} \in[0,1), \bar{z}_{a} \in[0,1)$ and $\bar{d} \geq 0$ be given. The system (7), under a given feedback scheme, is $\mathrm{m}$-th moment (robustly) stable provided that the following holds:

$$
\begin{cases}\lim _{k \rightarrow \infty} \mathcal{E}\left[\overline{\mathbf{x}}(k)^{m}\right]=0 & \text { if } \bar{z}_{f}=\bar{d}=0 \\ \exists b \in \mathbb{R}_{+} \text {s.t. } \lim _{\sup _{k \rightarrow \infty}} \mathcal{E}\left[\overline{\mathbf{x}}(k)^{m}\right]<b & \text { otherwise }\end{cases}
$$

The first limit in (13) is an internal stability condition while the second establishes external stability. The constant $b$ must be such that $\limsup _{k \rightarrow \infty} \mathcal{E}\left[\overline{\mathbf{x}}(k)^{m}\right]<b$ holds for all allowable perturbations $\mathbf{z}_{a}$ and $\mathbf{z}_{f}=G_{f}(\mathbf{x})$ satisfying (5)-(6).

\section{F. Overview of the Main Results and Conclusions}

The main results of this paper are the sufficiency theorems 3.2 and 3.4 proven in section III as well as the necessary condition of theorem 4.1 in section IV. The sufficiency conditions are proven constructively by means of the stabilizing feedback scheme of definition 3.2.

Such results lead to the conclusion that the limitations created by randomness in the plant and the link are mathematically equivalent. In addition, we find that the higher $\mathcal{C}$ the larger the tolerance to uncertainty in the plant.

\section{SUFFICIENCY CONDITIONS FOR THE EXISTENCE OF STABILIZING FEEDBACK SCHEMES}

In this section we derive constructive sufficient conditions for the existence of a stabilizing feedback scheme. We start with the deterministic case in subsection III-A, while III-B deals with random $\mathbf{r}$ and $\mathbf{a}$.

The following definition introduces the main idea behind the construction of a stabilizing feedback scheme.

Definition 3.1: (Upper-bound Sequence) Let $\bar{z}_{f} \in$ $[0,1), \bar{z}_{a} \in[0,1), \bar{d} \geq 0$ and $\varrho \in \mathbb{N}_{+} \bigcup\{\infty\}$ be given. Define the upper-bound sequence as:

$$
\begin{aligned}
\mathbf{v}(k+1)= & |\mathbf{a}(k)| 2^{-\mathbf{r}_{e}(k)} v(k)+ \\
& \bar{z}_{f} \max \{v(k-\varrho+1), \ldots, \mathbf{v}(k)\}+\bar{d},
\end{aligned}
$$

where $v(i)=0$ for $i<0, v(0)=\frac{1}{2}$ and $\mathbf{r}_{e}(k)$ is an effective rate given by:

$$
\mathbf{r}_{e}(k)=-\log \left(2^{-\mathbf{r}(k)}+\bar{z}_{a}\right)
$$

We adopt $v(0)=\frac{1}{2}$ to guarantee that $|x(0)| \leq v(0)$. If $x(0)=0$ then we can select $v(0)=0$. Notice that the multiplicative uncertainty $\bar{z}_{a}$ acts by reducing the effective rate $\mathbf{r}_{e}(k)$. After inspecting (15), we find that $\mathbf{r}_{e}(k) \leq$ $\min \left\{\mathbf{r}(k),-\log \left(\bar{z}_{a}\right)\right\}$.

Definition 3.2: (Stabilizing feedback scheme) We make use of the sequence specified in definition 3.1. Notice that $\mathbf{v}(k)$ can be constructed at the controller and the encoder because both have access to $\varrho, \bar{z}_{f}, \bar{z}_{a}, \bar{d}, \mathbf{r}(k-1)$ and $\mathbf{a}(k-1)$.

The feedback scheme is defined as:

- Encoder: Measures $x(k)$ and computes $b_{i} \in\{0,1\}$ such that:

$$
\left(b_{1}, \ldots, b_{\bar{r}}\right)=\arg \max _{\sum_{i=1}^{\bar{r}} b_{i} \frac{1}{2^{i}} \leq\left(\frac{\mathbf{x}(k)}{2 \mathbf{v}(k)}+\frac{1}{2}\right)} \sum_{i=1}^{\bar{r}} b_{i} \frac{1}{2^{i}}
$$


Place $\left(b_{1}, \ldots, b_{\bar{r}}\right)$ for transmission. For any $r(k) \in$ $\left\{r_{\text {min }}, \ldots, \bar{r}\right\}$, the above construction provides a centroid approximation for $x(k) \in[-v(k), v(k)]$, satisfying

$$
\left|x(k)-2 v(k)\left(\sum_{i=1}^{r(k)} b_{i} \frac{1}{2^{i}}+\frac{1}{2^{r(k)+1}}-\frac{1}{2}\right)\right| \leq 2^{-r(k)} v(k)
$$

- Controller: From the $\bar{r}$ bits placed for transmission in the stochastic link, only $\mathbf{r}(k)$ bits go through. Compute $\mathbf{u}(k)$ as:

$$
\mathbf{u}(k)=-\mathbf{a}(k) 2 v(k)\left(\sum_{i=1}^{\mathbf{r}(k)} b_{i} \frac{1}{2^{i}}+\frac{1}{2^{\mathbf{r}(k)+1}}-\frac{1}{2}\right)
$$

As expected, the transmission of state information through a finite capacity medium requires quantization. The encoding scheme of definition 3.2 is not an exception and is structurally identical to the ones used in [1], [18].

The following lemma suggests that, in the construction of stabilizing controllers, we may choose to focus on the dynamics of the sequence $v(k)$. That simplifies the analysis in the presence of uncertainty because the dynamics of $v(k)$ is described by a first-order difference equation.

Lemma 3.1: Let $\bar{z}_{f} \in[0,1), \bar{z}_{a} \in[0,1)$ and $\bar{d} \geq 0$ be given. If $\mathbf{x}(k)$ is the solution of (7) under the feedback scheme of definition 3.2, then the following holds:

$$
\overline{\mathbf{x}}(k) \leq \mathbf{v}(k)
$$

for all $\varrho \in \mathbb{N}_{+} \bigcup\{\infty\}$, every choice $G_{f} \in \Delta_{f, \varrho}$ and $\left|\mathbf{z}_{\mathbf{a}}(k)\right| \leq \bar{z}_{a}$, where

$$
\Delta_{f, \varrho}=\left\{G_{f}: \mathbb{R}^{\infty} \rightarrow \mathbb{R}^{\infty}:\left\|G_{f}\right\|_{\infty(\varrho)} \leq \bar{z}_{f}\right\}
$$

Proof: We proceed by induction, assuming that $\bar{x}(i) \leq v(i)$ for $i \in\{0, \ldots, k\}$ and proving that $\bar{x}(k+1) \leq v(k+1)$. From (7), we get:

$$
\begin{aligned}
|x(k+1)| \leq & |a(k)|\left|x(k)+\frac{u(k)}{a(k)}\right|+ \\
& \left|z_{a}(k)\right||a(k)||x(k)|+\left|z_{f}(k)\right|+|d(k)|
\end{aligned}
$$

The way the encoder constructs the binary expansion of the state, as well as (17), allow us to conclude that

$$
\left|x(k)+\frac{u(k)}{a(k)}\right| \leq 2^{-r(k)} v(k)
$$

Now we recall that $\left|z_{a}(k)\right| \leq \bar{z}_{a},\left|z_{f}(k)\right| \leq \bar{z}_{f} \max \{v(k-$ $\varrho+1), \ldots, v(k)\}$ and that $|d(k)| \leq \bar{d}$, so that (19) implies:

$$
\begin{aligned}
|x(k+1)| \leq & |a(k)|\left(2^{-r(k)}+\bar{z}_{a}\right) v(k)+ \\
& \bar{z}_{f} \max \{v(k-\varrho+1), \ldots, \mathbf{v}(k)\}+\bar{d}
\end{aligned}
$$

The proof is concluded once we realize that $|x(0)| \leq v(0)$.

\section{A. The Deterministic Case}

We start by deriving a sufficient condition for the existence of a stabilizing feedback scheme in the deterministic case, i.e., $\mathbf{r}(k)=\mathcal{C}$ and $\log (|\mathbf{a}(k)|)=\mathcal{R}$. Subsequently, we move for the stochastic case where we derive a sufficient condition for stabilizability.

Theorem 3.2: (Sufficiency conditions for Robust Stability) Let $\varrho \in \mathbb{N}_{+} \bigcup\{\infty\}, \bar{z}_{f} \in[0,1), \bar{z}_{a} \in[0,1)$ and $\bar{d} \geq 0$ be given and $h(k)$ be defined as

$$
h(k)=2^{k\left(\mathcal{R}-\mathcal{C}_{e}\right)}, k \geq 0
$$

where $\mathcal{C}_{e}=r_{e}=-\log \left(2^{-\mathcal{C}}+\bar{z}_{a}\right)$.

Consider that $\mathbf{x}(k)$ is the solution of (7) under the feedback scheme of definition 3.2 as well as the following conditions:

- (C 1) $\mathcal{C}_{e}>\mathcal{R}$

- (C 2) $\bar{z}_{f}\|h\|_{1}<1$

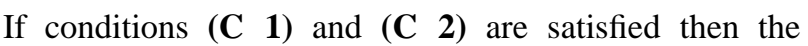
following holds for all $|\mathbf{d}(t)| \leq \bar{d}, G_{f} \in \Delta_{f, \varrho}$ and $\left|\mathbf{z}_{a}(k)\right| \leq$ $\bar{z}_{a}$ :

$$
\bar{x}(k) \leq\|h\|_{1}\left(\bar{z}_{f} \frac{\|h\|_{1} \bar{d}+\frac{1}{2}}{1-\|h\|_{1} \bar{z}_{f}}+\bar{d}\right)+h(k) \frac{1}{2}
$$

where $\Delta_{f, \varrho}$ is given by:

$$
\Delta_{f, \varrho}=\left\{G_{f}: \mathbb{R}^{\infty} \rightarrow \mathbb{R}^{\infty}:\left\|G_{f}\right\|_{\infty(\varrho)} \leq \bar{z}_{f}\right\}
$$

Proof: From definition 3.1, we know that, for arbitrary $\varrho \in$ $\mathbb{N}_{+} \cup\{\infty\}$, the following is true:

$v(k+1)=2^{\mathcal{R}-\mathcal{C}_{e}} v(k)+\bar{z}_{f} \max \{v(k-\varrho+1), \ldots, \mathbf{v}(k)\}+\bar{d}$

Solving the difference equation gives:

$$
\begin{aligned}
& v(k)=2^{k\left(\mathcal{R}-\mathcal{C}_{e}\right)} v(0)+\sum_{i=0}^{k-1} \\
& 2^{(k-i-1)\left(\mathcal{R}-\mathcal{C}_{e}\right)}\left(\bar{z}_{f} \max \{v(i-\varrho+1), \ldots, \mathbf{v}(i)\}+\bar{d}\right)
\end{aligned}
$$

which, using $\left\|\Pi_{k} v\right\|_{\infty}=\max \{v(0), \ldots, \mathbf{v}(k)\}$, leads to:

$$
v(k) \leq\|h\|_{1}\left(\bar{z}_{f}\left\|\Pi_{k} v\right\|_{\infty}+\bar{d}\right)+2^{k\left(\mathcal{R}-\mathcal{C}_{e}\right)} v(0)
$$

But we also know that $2^{k\left(\mathcal{R}-\mathcal{C}_{e}\right)}$ is a decreasing function of $k$, so that:

$$
\left\|\Pi_{k} v\right\|_{\infty} \leq\|h\|_{1}\left(\bar{z}_{f}\left\|\Pi_{k} v\right\|_{\infty}+\bar{d}\right)+v(0)
$$

which implies:

$$
\left\|\Pi_{k} v\right\|_{\infty} \leq \frac{\|h\|_{1} \bar{d}+v(0)}{1-\|h\|_{1} \bar{z}_{f}}
$$

Direct substitution of (27) in (25) leads to:

$$
v(k) \leq\|h\|_{1}\left(\bar{z}_{f} \frac{\|h\|_{1} \bar{d}+v(0)}{1-\|h\|_{1} \bar{z}_{f}}+\bar{d}\right)+2^{k\left(\mathcal{R}-\mathcal{C}_{e}\right)} v(0)
$$

The proof is complete once we make $v(0)=\frac{1}{2}$ and use lemma 3.1 to conclude that $\bar{x}(k) \leq v(k)$. 


\section{B. Sufficient Condition for the Stochastic Case}

The following lemma provides a sequence, denoted by $v_{m}(k)$, which is an upper-bound for the m-th moment of $\overline{\mathbf{x}}(k)$. We show that $v_{m}$ is propagated according to a firstorder difference equation that is suitable for the analysis in the presence of uncertainty.

Lemma 3.3: (M-th moment boundedness) Let $\varrho \in \mathbb{N}_{+}$, $\bar{z}_{f} \in[0,1), \bar{z}_{a} \in[0,1)$ and $\bar{d} \geq 0$ be given along with the following set:

$$
\Delta_{f, \varrho}=\left\{G_{f}: \mathbb{R}^{\infty} \rightarrow \mathbb{R}^{\infty}:\left\|G_{f}\right\|_{\infty(\varrho)} \leq \bar{z}_{f}\right\}
$$

Given $m$, consider the following sequence:

$$
\begin{aligned}
& v_{m}(k)=h_{m}(k) v_{m}(0)+\sum_{i=0}^{k-1} h_{m}(k-i-1) \\
& \left(\varrho^{\frac{1}{m}} \bar{z}_{f} \max \left\{v_{m}(i-\varrho+1), \ldots, v_{m}(i)\right\}+\bar{d}\right)
\end{aligned}
$$

where $v_{m}(i)=0$ for $i<0, v_{m}(0)=\frac{1}{2}, h_{m}(k)$ is the impulse response given by:

$$
h_{m}(k)=\left(\mathcal{E}\left[2^{m\left(\log (|a(k)|)-r_{e}(k)\right)}\right]\right)^{\frac{k}{m}}, k \geq 0
$$

and $r_{e}(k)=-\log \left(2^{-r(k)}+\bar{z}_{a}\right)$. If $\mathbf{x}(k)$ is the solution of (7) under the feedback scheme of definition 3.2, then the following holds

$$
\mathcal{E}\left[\overline{\mathbf{x}}(k)^{m}\right] \leq v_{m}(k)^{m}
$$

for all $|\mathbf{d}(t)| \leq \bar{d}, G_{f} \in \Delta_{f, \varrho}$ and $\left|\mathbf{z}_{a}(k)\right| \leq \bar{z}_{a}$.

Proof: Since lemma 3.1 guarantees that $\bar{x}(k+1) \leq v(k+$ $1)$, we only need to show that $\mathcal{E}\left[\mathbf{v}(k+1)^{m}\right]^{\frac{1}{m}} \leq v_{m}(k+1)$. Again, we proceed by induction by noticing that $v(0)=$ $v_{m}(0)$ and by assuming that $\mathcal{E}\left[\mathbf{v}(i)^{m}\right]^{\frac{1}{m}} \leq v_{m}(i)$ for $i \in$ $\{1, \ldots, k\}$. From definition 3.1, we know that:

$$
\begin{aligned}
& \mathcal{E}\left[\mathbf{v}(k+1)^{m}\right]^{\frac{1}{m}}=\mathcal{E}\left[\left(2^{\log (|\mathbf{a}(k)|)-\mathbf{r}_{e}(k)} \mathbf{v}(k)+\right.\right. \\
&\left.\left.\bar{z}_{f} \max \{\mathbf{v}(j-\varrho+1), \ldots, \mathbf{v}(j)\}+\bar{d}\right)^{m}\right]^{\frac{1}{m}}
\end{aligned}
$$

Using Minkowsky's inequality [6] as well as the fact that $\mathbf{v}(i)$ is independent of $\mathbf{a}(j)$ and $\mathbf{r}_{e}(j)$ for $j \geq i$, we get:

$$
\begin{array}{r}
\mathcal{E}\left[\mathbf{v}(k+1)^{m}\right]^{\frac{1}{m}} \leq \mathcal{E}\left[2^{m\left(\log (|\mathbf{a}(k)|)-\mathbf{r}_{e}(k)\right)}\right]^{\frac{1}{m}} \mathcal{E}\left[\mathbf{v}(k)^{m}\right]^{\frac{1}{m}}+ \\
\bar{z}_{f} \mathcal{E}\left[\max \{\mathbf{v}(k-\varrho+1), \ldots, \mathbf{v}(k)\}^{m}\right]^{\frac{1}{m}}+\bar{d}
\end{array}
$$

which, using the inductive assumption, implies the following inequality:

$$
\begin{gathered}
\mathcal{E}\left[\mathbf{v}(k+1)^{m}\right]^{\frac{1}{m}} \leq \mathcal{E}\left[2^{m\left(\log (|\mathbf{a}(k)|)-\mathbf{r}_{e}(k)\right)}\right]^{\frac{1}{m}} v_{m}(k)+ \\
\varrho^{\frac{1}{m}} \bar{z}_{f} \max \left\{v_{m}(k-\varrho+1), \ldots, v_{m}(k)\right\}+\bar{d}
\end{gathered}
$$

where we used the fact that, for arbitrary random variables $\mathbf{s}_{1}, \ldots, \mathbf{s}_{n}$, the following holds:

$$
\begin{aligned}
& \mathcal{E}\left[\max \left\{\left|\mathbf{s}_{1}\right|, \ldots,\left|\mathbf{s}_{n}\right|\right\}^{m}\right] \leq \mathcal{E}\left[\sum_{i=1}^{n}\left|\mathbf{s}_{i}\right|^{m}\right] \leq \\
& n \max \left\{\mathcal{E}\left[\left|\mathbf{s}_{1}\right|^{m}\right], \ldots, \mathcal{E}\left[\left|\mathbf{s}_{n}\right|^{m}\right]\right\}
\end{aligned}
$$

The proof follows once we notice that the right hand side of (34) is just $v_{m}(k+1)$.

Theorem 3.4: (Sufficient Condition) Let $m, \varrho \in \mathbb{N}_{+}$, $\bar{z}_{f} \in[0,1), \bar{z}_{a} \in[0,1)$ and $\bar{d} \geq 0$ be given along with the quantities bellow:

$$
\begin{gathered}
\beta(m)=\frac{1}{m} \log \mathcal{E}\left[2^{m \mathbf{l}^{\delta}(k)}\right] \\
h_{m}(k)=2^{k\left(\mathcal{R}+\beta(m)+\alpha_{e}(m)-\mathcal{C}_{e}\right)}, k \geq 0
\end{gathered}
$$

where

$$
\begin{gathered}
r_{e}(k)=-\log \left(2^{-r(k)}+\bar{z}_{a}\right)=\mathcal{C}_{e}-\mathbf{r}_{e}^{\delta}(k) \\
\alpha_{e}(m)=\frac{1}{m} \log \left(\mathcal{E}\left[2^{m \mathbf{r}_{e}^{\delta}(k)}\right]\right)
\end{gathered}
$$

and $^{1} \mathbf{r}_{e}^{\delta}(k)$ is a zero-mean i.i.d. process. Consider that $\mathbf{x}(k)$ is the solution of (7) under the feedback scheme of definition 3.2 as well as the following conditions:

- (C 3) $\mathcal{C}_{e}>\mathcal{R}+\beta(m)+\alpha_{e}(m)$

- (C 4) $\varrho^{\frac{1}{m}} \bar{z}_{f}\left\|h_{m}\right\|_{1}<1$

If conditions (C 3) and (C 4) are satisfied, then the following holds for all $|\mathbf{d}(t)| \leq \bar{d}, G_{f} \in \Delta_{f, \varrho}$ and $\left|\mathbf{z}_{a}(k)\right| \leq$ $\bar{z}_{a}$ :

$$
\begin{array}{r}
\mathcal{E}\left[\overline{\mathbf{x}}(k)^{m}\right]^{\frac{1}{m}} \leq\left\|h_{m}\right\|_{1}\left(\varrho^{\frac{1}{m}} \bar{z}_{f} \frac{\left\|h_{m}\right\|_{1} \bar{d}+\frac{1}{2}}{1-\varrho^{\frac{1}{m}} \bar{z}_{f}\left\|h_{m}\right\|_{1}}+\bar{d}\right)+ \\
h_{m}(k) \frac{1}{2}
\end{array}
$$

where $\Delta_{f, \varrho}$ is given by:

$$
\Delta_{f, \varrho}=\left\{G_{f}: \mathbb{R}^{\infty} \rightarrow \mathbb{R}^{\infty}:\left\|G_{f}\right\|_{\infty(\varrho)} \leq \bar{z}_{f}\right\}
$$

Proof: Using $v_{m}$ from lemma 3.3, we arrive at:

$$
v_{m}(k) \leq h_{m}(k) v_{m}(0)+\left\|h_{m}\right\|_{1}\left(\varrho^{\frac{1}{m}} \bar{z}_{f}\left\|\Pi_{k} v_{m}\right\|_{\infty}+\bar{d}\right)
$$

where we use $\left\|\Pi_{k} v_{m}\right\|_{\infty}=\max \left\{v_{m}(0), \ldots, v_{m}(k)\right\}$. But from (38), we conclude that:

$$
\left\|\Pi_{k} v_{m}\right\|_{\infty} \leq v_{m}(0)+\left\|h_{m}\right\|_{1}\left(\varrho^{\frac{1}{m}} \bar{z}_{f}\left\|\Pi_{k} v_{m}\right\|_{\infty}+\bar{d}\right)
$$

or equivalently:

$$
\left\|\Pi_{k} v_{m}\right\|_{\infty} \leq \frac{v_{m}(0)+\left\|h_{m}\right\|_{1} \bar{d}}{1-\left\|h_{m}\right\|_{1} \varrho^{\frac{1}{m}} \bar{z}_{f}}
$$

Substituting (40) in (38), gives:

$$
\begin{aligned}
& v_{m}(k) \leq h_{m}(k) v_{m}(0)+ \\
& \quad\left\|h_{m}\right\|_{1}\left(\varrho^{\frac{1}{m}} \bar{z}_{f} \frac{v_{m}(0)+\left\|h_{m}\right\|_{1} \bar{d}}{1-\left\|h_{m}\right\|_{1} \varrho^{\frac{1}{m}} \bar{z}_{f}}+\bar{d}\right)
\end{aligned}
$$

\footnotetext{
${ }^{1}$ Notice that if $\bar{z}_{a}=0$ then $C_{e}=C$ and $\alpha_{e}(m)=\alpha(m)$.
} 
The proof follows from lemma 3.3 and by noticing that $h_{m}(k)$ can be rewritten as:

$$
\begin{aligned}
& h_{m}(k)= \\
& \quad\left(\mathcal{E}\left[2^{m\left(\log (|\mathbf{a}(k)|)-\mathbf{r}_{e}(k)\right)}\right)^{\frac{k}{m}}=2^{k\left(\mathcal{R}+\beta(m)+\alpha_{e}(m)-\mathcal{C}_{e}\right)}\right.
\end{aligned}
$$

\section{NECESSARY CONDITION FOR THE EXISTENCE OF STABILIZING FEEDBACK SCHEMES}

Consider that $\bar{z}_{a}=\bar{z}_{f}=\bar{d}=0$. We derive necessary conditions for the existence of an internally stabilizing feedback scheme. We emphasize that the proofs in this section use the m-th moment stability as a stability criteria and that they are valid regardless of the encoding/decoding scheme. They follow from a counting argument ${ }^{2}$ which is identical to the one used by [18]. Necessary conditions for stability were also studied for the Gaussian channel in [19] and for other stochastic channels in [15], [16]. A necessary condition for the almost sure stability for general stochastic channels is given by [17]. We include our treatment, because it provides necessary conditions for $\mathrm{m}$-th moment stability, which are inequalities involving directly the defined quantities $\alpha(m)$ and $\beta(m)$. In section III-B, we show, for first order systems, that the necessary condition of Theorem 4.1 is not conservative. In [11], we prove that Theorem 4.1 is not conservative if $B=I$.

We derive the necessary condition for the following class of state-space representations:

$$
\mathbf{x}(k)=\mathbf{U}(k) \mathbf{x}(k)+B \mathbf{u}(k)
$$

where $x(k) \in \mathbb{R}^{n}, u(k) \in \mathbb{R}^{n_{b}}, B \in \mathbb{R}^{n \times n_{b}}$ and $\mathbf{U}(k)$ is a block upper-triangular matrix of the form:

$\mathbf{U}(k)=\left[\begin{array}{cccc}\mathbf{a}(k) \operatorname{Rot}(k) & \cdots & \cdots & \\ 0 & \mathbf{a}(k) \operatorname{Rot}(k) & \ddots & \\ \vdots & \ddots & & \vdots \\ 0 & \cdots & 0 & \mathbf{a}(k) \operatorname{Rot}(k)\end{array}\right]$

and Rot is a sequence of random rotation matrices satisfying $\operatorname{det}(\operatorname{Rot}(k))=1$. We also assume that $\operatorname{Rot}$ is independent of $\mathbf{r}$.

Theorem 4.1: Let $\mathbf{x}(k)$ be the solution of the state-space equation (43) along with $\alpha(m)$ and $\beta(m)$ given by:

$$
\begin{aligned}
& \alpha(m)=\frac{1}{m} \log \left(E\left[2^{m \mathbf{r}^{\delta}(k)}\right]\right) \\
& \beta(m)=\frac{1}{m} \log \left(E\left[2^{m \mathbf{l}^{\delta}(k)}\right]\right)
\end{aligned}
$$

\footnotetext{
${ }^{2}$ We also emphasize that this proof is different from what we had originally. The present argument was suggested by a reviewer of one of our publications
}

and the norm on the vector $x(k)$ be represented as:

$$
\|x(k)\|_{\infty}=\max _{i}\left|[x(k)]_{i}\right|,
$$

where $[x(k)]_{i}$ are components of the vector $x(k)$.

If the state satisfies the following:

$$
\sup _{k} E\left[\sup _{x(0) \in[-1 / 2,1 / 2]^{n}}\|\mathbf{x}(k)\|_{\infty}^{m}\right]<\infty
$$

then the following must hold:

$$
C-\alpha\left(\frac{m}{n}\right) \geq n \beta(m)+n R
$$

Proof: Consider a specific realization of Rot, $\mathbf{r}$ and $\mathbf{a}$ along with the following sets:

$$
\begin{array}{r}
\bar{\Omega}_{k}=\left\{\Pi_{i=0}^{k-1} U(i) x(0): x(0) \in[-1 / 2,1 / 2]^{n}\right\} \\
\Omega_{k}\left(\{u(i)\}_{i=0}^{k-1}\right)=\left\{x(k): x(0) \in[-1 / 2,1 / 2]^{n},\right. \\
\left.\{u(i)\}_{i=0}^{k-1}=\mathcal{F}(x(0), k)\right\}
\end{array}
$$

where $u(k)$ is obtained through a fixed feedback law $\mathcal{F}(x(0), k)$. Since $x(k)$ is given by (43) and $\{u(i)\}_{i=0}^{k-1}$ can take, at most, $2^{\sum_{i=0}^{k-1} r(i)}$ values, we find that:

$$
\frac{\operatorname{Vol}\left(\bar{\Omega}_{k}\right)}{\max _{\{u(i)\}_{i=0}^{k-1}} \operatorname{Vol}\left(\Omega_{k}\left(\{u(i)\}_{i=0}^{k-1}\right)\right)} \leq 2^{\sum_{i=0}^{k-1} r(i)}
$$

Computing bounds for the volumes, we get:

$$
\begin{aligned}
& \operatorname{Vol}\left(\bar{\Omega}_{k}\right)=2^{\sum_{i=0}^{k-1} \log |\operatorname{det}(U(i))|} \\
& \operatorname{Vol}\left(\Omega_{k}\left(\{u(i)\}_{i=0}^{k-1}\right)\right) \leq v^{n}(k)
\end{aligned}
$$

where $v(k)=2 \sup _{x(0) \in[-1 / 2,1 / 2]^{n}}\|x(k)\|_{\infty}$. Consequently, using (52) we infer that:

$$
2^{\sum_{i=0}^{k-1} \log |\operatorname{det}(U(i))|} 2^{-\sum_{i=0}^{k-1} r(i)} \leq 2^{n} v^{n}(k)
$$

By taking expectations, the m-th moment stability assumption leads to:

$$
\begin{gathered}
\limsup _{k \rightarrow \infty}\left(\mathcal{E}\left[2^{\frac{m}{n} \log |\operatorname{det}(\mathbf{U}(k))|}\right] \mathcal{E}\left[2^{-\frac{m}{n} \mathbf{r}(k)}\right]\right)^{k} \leq \\
2^{m} \limsup _{k \rightarrow \infty} \mathcal{E}\left[\mathbf{v}^{m}(k)\right]<\infty
\end{gathered}
$$

which implies that:

$$
C>\alpha\left(\frac{m}{n}\right)+n \beta(m)+n R
$$

where we used the fact that $\mathcal{E}\left[2^{\frac{m}{n} \log |\operatorname{det}(\mathbf{U}(k))|}\right] \mathcal{E}\left[2^{-\frac{m}{n} \mathbf{r}(k)}\right]<1$ must hold and that $\log |\operatorname{det}(\mathbf{U}(k))|=n \log |\mathbf{a}(k)|$.

Corollary 4.2: Let $\mathbf{x}(k)$ be the solution of the following linear and time-invariant system:

$$
\mathbf{x}(k+1)=A \mathbf{x}(k)+B \mathbf{u}(k)
$$

If the state satisfies the following:

$$
\sup _{k} E\left[\sup _{x(0) \in[-1 / 2,1 / 2]^{n}}\|\mathbf{x}(k)\|_{\infty}^{m}\right]<\infty
$$


then the following must hold:

$$
C-\alpha\left(\frac{m}{n_{\text {unstable }}}\right) \geq \sum_{i=1}^{n} \max \left\{\log \left|\lambda_{i}(A)\right|, 0\right\}
$$

where $n_{\text {unstable }}$ is the number of unstable eigenvalues.

Proof: The proof is a direct adaptation of the proof of Theorem 4.1.

\section{PROPERTIES OF $\alpha(m)$ AND $\beta(m)$}

The following are properties of $\alpha(m)$ and $\beta(m)$ :

- Note that Jensen's inequality implies that $\alpha(m) \geq 0$ and $\beta(m) \geq 0$, where equality is achieved only if the corresponding random variable is deterministic.

- by means of a Taylor expansion and taking limits, we get

$$
\lim _{m \searrow 0} \alpha(m)=\lim _{m \searrow 0} \beta(m)=0
$$

- the opposite limiting case, gives

$$
\begin{gathered}
\lim _{m \rightarrow \infty} \alpha(m)=C-r_{\text {min }} \\
\lim _{m \rightarrow \infty} \beta(m)=\log \left(a^{\text {sup }}\right)-R
\end{gathered}
$$

where

$$
\begin{gathered}
r_{\text {min }}=\min \{r \in\{0, \ldots, \bar{r}\}: \mathcal{P}(\mathbf{r}(k)=r) \neq 0\} \\
a^{\text {sup }}=\sup \{\tilde{a}: \mathcal{P}(|\mathbf{a}(k)| \geq \tilde{a}) \neq 0\}
\end{gathered}
$$

- $\alpha(m)$ and $\beta(m)$ are non-decreasing functions of $m$

Further properties of $\alpha(m)$ and $\beta(m)$ as well as more conclusions can be found in [11]. With a specific importance in the stabilization of time-invariant linearizable systems, we may conclude that the following is a necessary condition for stability in the sense of Lyapunov (i.s.L.):

$$
r_{\text {min }} \geq \sum_{i} \max \left\{0, \log \left|\lambda_{i}(A)\right|\right\}
$$

where $A$ is the dynamic matrix of the linearized system. Notice that (64) follows from (62) by noticing that stability i.s.L. implies $\mathrm{m}$-th moment stability for all $\mathrm{m}$. This is important because it suggests that a deterministic link must always be present. In addition $r_{\min }>\sum_{i} \max \left\{0, \log \left|\lambda_{i}(A)\right|\right\}$ is also sufficient [18]. Consequently, we conclude that studying the stabilizability i.s.L. for deterministic links is sufficiently general.

\section{ACKNOWLEDGMENTS}

The authors would like to thank Prof. Sekhar Tatikonda (Yale University) for providing pre-print papers comprising some of his most recent results. We also would like to thank Prof. Sanjoy Mitter (M.I.T.) for giving valuable motivation. This work was sponsored by UCLA, MURI project award: 0205-G-CB222. Nuno C. Martins was partially supported by the Portuguese Foundation for Science and Technology and the European Social Fund, PRAXIS BD19630/99. Nicola Elia has been supported by NSF under the Career Award grant number ECS-0093950.

\section{REFERENCES}

[1] Brockett, R.W.;Liberzon, D. "Quantized Feedback Stabilization of Linear Systems", IEEE Transactions on Automatic Control, vol 45, pp. 1279-1289, July 2000

[2] Cover, T.M; Thomas, J. A.; "Elements of Information Theory", Wiley-Iterscience Publication, 1991

[3] Elia, N., "Control-Oriented feedback communication schemes", Proceedings of the IEEE Conference on Decision and Control, 2003

[4] Elia, N., "Stabilization of systems with erasure actuation and sensory channels', Proc. 40th Allerton Conference 2002.

[5] Elia, N., "Stabilization in the presence of fading channels", Proc. of American Control Conference 2003.

[6] Halmos, P. R.; “ Measure Theory”, Springer Verlag, 1974

[7] Jain, R., Simsek, T., Varayia P. " Control under Communication Constraints", IEEE CDC2002, Vol. 5, pp. 3209-15, 2002

[8] Liberzon, D.; "On Stabilization of Linear Systems With Limited Information", IEEE Transactions on Automation and Control, Vol 48(2), pp. 304-7, 2003

[9] Li, K.; Baillieul, J., "Robust Quantization for Digital Finite Communication Bandwidth (DFCB) Control", Proceedings of the IEEE Conference on Decision and Control, 2003

[10] Li, X.; Wong, W. S., " State Estimation with Communication Constraints", Systems and Control Letters 28 (1996), pp. 49-54

[11] Martins, N. C. "Information Theoretic Aspects of the Control and Mode Estimation of Stochastic Systems", MIT- Ph.D. Thesis, 2004

[12] Martins, N. C.; Dahleh, M. A.;Elia N., "Stabilization of Uncertain Systems in the Presence of Finite Data-Rate Feedback",IFAC-Nolcos 2004, Stuttgart, Germany

[13] Nair, G.; Dey S.; Evans, R.J.; "Infimum Data Rates for Stabilizing Markov Jump Linear Systems", Proc. IEEE Conference on Decision and Control, 2003, pp. 1176-81

[14] Nair, G. N. and Evans, R. J., "Stabilization with Data-Rate-Limited Feedback: Tightest Attainable Bounds." Systems and Control Letters, Vol 41, pp. 49-76, 2000

[15] Sahai, A.; "Evaluating Channels for Control: Capacity Reconsidered", Proc. ACC., pp. 2358 - 2362, 2000

[16] Sahai, A.; “Anytime Information Theory”, Ph.D. Thesis, M.I.T. 2001

[17] Tatikonda, S.; "Control under Communication Constraints: Part I and II", submitted to the IEEE Transactions on Automatic Control

[18] Tatikonda, S.; "Control under Communication Constraints", Ph.D. Thesis, M.I.T. 2000

[19] Tatikonda, S.; Sahai, A.; Mitter, S. K. "Control of LQG Systems under Communication Constraints", Proc. IEEE Conference on Decision and Control, 1998

[20] Witsenhausen, H., "Separation of Estimation and Control for Discrete-Time Systems", Proceeding of the IEEE, Volume 59, No 11, November 1971

[21] Wong, W.S.; Brockett, R.W.; "Systems with finite communication bandwidth constraints -I: State estimation problems" IEEE Trans. Automat. Control, Vol 42, pp. 1294-1298, Sept 1997

[22] Wong, W.S.; Brockett, R.W.; "Systems with finite communication bandwidth constraints -II: Stabilization with Limited Information Feedback" IEEE Trans. Automat. Control, Vol 44, No. 5 pp. 10491053, 1999 